\title{
ON THE DIMENSION OF A GRAPH
}

\section{Paul Erdös, Frank Harary and William T. Tutte}

Our purpose in this note is to present a natural geometrical definition of the dimension of a graph and to explore some of its ramifications. In $\S 1$ we determine the dimension of some special graphs. We observe in $\$ 2$ that several results in the literature are unified by the concept of the dimension of a graph, and state some related unsolved problems.

We define the dimension of a graph $G$, denoted $\operatorname{dim} G$, as the minimum number $n$ such that $G$ can be embedded into Euclidean $n$-space $E_{n}$ with every edge of $G$ having length 1 . The vertices of $G$ are mapped onto distinct points of $E_{n}$, but there is no restriction on the crossing of edges.

1. Some graphs and their dimensions. Let $K_{n}$ be the complete graph with $n$ vertices in which every pair of vertices are adjacent (joined by an edge). The triangle $K_{3}$ and the tetrahedron $K_{4}$ are shown in Figure 1 .
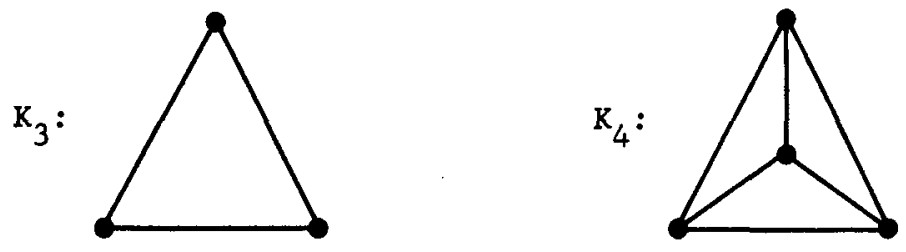

Fig. 1.

The dimension of $K_{3}$ is 2 since it may be drawn as a unit equilateral triangle. But clearly, $\operatorname{dim} K_{4}=3$ and in general $\operatorname{dim} K_{n}=n-1$.

By $K_{n}-x$ we mean the graph obtained from the complete graph $K_{n}$ by deleting any one edge, $x$. For example $K_{3}-x$ and $K_{4}-x$ are shown in Figure 2.
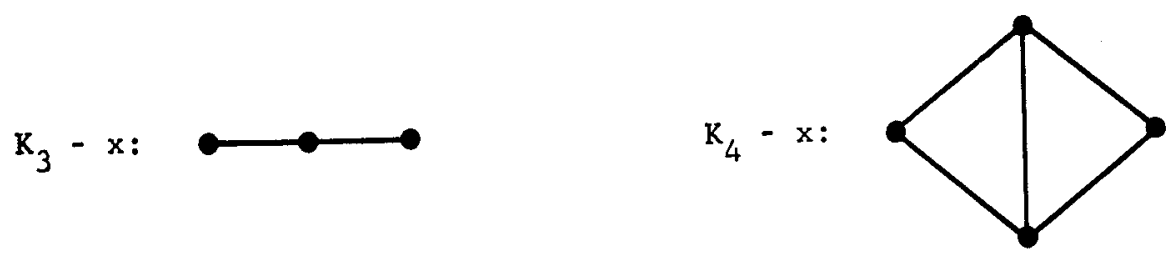

Fig. 2.

From this figure, we see at once that $\operatorname{dim}\left(K_{3}-x\right)=1$ and that $\operatorname{dim}\left(K_{4}-x\right)=2$ since it can be drawn as two equilateral triangles with the same base. By a similar construction it is easy to show that in general $\operatorname{dim}\left(K_{n-1}-x\right)=n-2$.

The complete bicoloured graph $K_{m, n}$ has $m$ vertices of one colour, $n$ of another colour, and two vertices are adjacent if and only if they have 
different colours. We shall see how to determine the dimension of $K_{m, n}$ for all positive integers $m$ and $n$. In Figure 3 are shown three of these graphs, each of which we will see has a different dimension.
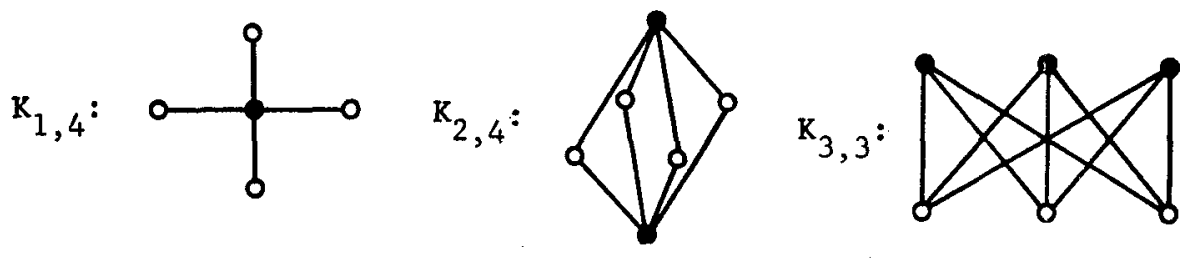

Fig. 3.

Which of the graphs $K_{n, n}$ have dimension 2 ? Since $K_{1,1}=K_{2}$, $\operatorname{dim} K_{1,1}=1$, and as shown in Figure $3, \operatorname{dim} K_{1,4}=2$. Obviously, for every $n>1, \operatorname{dim} K_{1, n}=2$. There is also one other complete bicoloured graph with dimension 2, namely the rhombus $K_{2,2}$. Again from the figure, we see that $\operatorname{dim} K_{2,4}=3$ and in general that $\operatorname{dim} K_{2, n}=3$ when $n \geqslant 3$. Finally, it is easy to show that the dimension of every other graph $K_{m, n}$ not already mentioned in this paragraph is 4 , including the famous 3 houses3 utilities graph $K_{3,3}$. The proof is due to Lenz, as mentioned in a paper by Erdös [2], and proceeds as follows.

Let $\left\{u_{i}\right\}$ be the $m$ vertices of the first colour and let $\left\{v_{j}\right\}$ be the $n$ vertices of the second colour. We assign coordinates in $E_{4}$ to $u_{i}=\left(x_{i}, y_{i}, 0,0\right)$ and $v_{j}=\left(0,0, z_{j}, w_{j}\right)$ in such a way that $x_{i}{ }^{2}+y_{i}{ }^{2}=\frac{1}{2}$ and $z_{j}{ }^{2}+w_{j}{ }^{2}=\frac{1}{2}$. Then every distance $d\left(u_{i}, v_{j}\right)=1$, proving the assertion.

In the next two illustrations of the dimension of a graph we use the operations of the "join" and the "product" of two graphs $G_{1}$ and $G_{2}$. Let $V_{1}$ and $V_{2}$ be their respective vertex sets. The join $G_{1}+G_{2}$ of two disjoint graphs contains both of them and also has an edge joining each vertex of $G_{1}$ with each vertex of $G_{2}$. The cartesian product $G_{1} \times G_{2}$ of $G_{1}$ and $G_{2}$ has $V_{1} \times V_{2}$ as its set of vertices. Two vertices $u=\left(u_{1}, u_{2}\right)$ and $v=\left(v_{1}, v_{2}\right)$ are adjacent in $G_{1} \times G_{2}$ if and only if $u_{1}=v_{1}$ and $u_{2} v_{2}$ is an edge of $G_{2}$ or $u_{2}=v_{2}$ and $u_{1} v_{1}$ is in $G_{1}$. Let $P_{n}$ denote the polygon with $n$ sides. By the wheel with $n$ spokes is meant the graph $P_{n}+K_{1}$; see Figure 4,

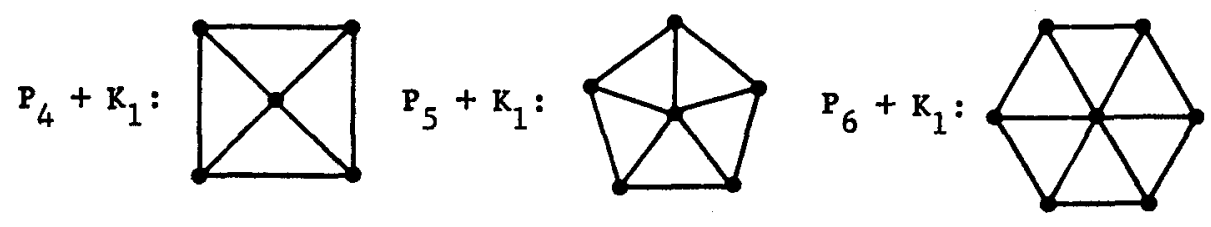

Fig. 4.

What is the dimension of a wheel? We already have one example since the smallest wheel $P_{3}+K_{1}=K_{4}$ has dimension 3. From Figure 4, we see that $\operatorname{dim}\left(P_{4}+K_{1}\right)=\operatorname{dim}\left(P_{5}+K_{1}\right)=3$ and that $\operatorname{dim}\left(P_{6}+K_{1}\right)=2$. By making 
expeditious use of the unit sphere, the reader can verify that for all $n>6$, $\operatorname{dim}\left(P_{n}+K_{1}\right)=3$. Thus we observe that the dimension of the $n$-spoked wheel is 3 except for "the odd number 6 ".

The $n$-cube $Q_{n}$ is defined as the cartesian product of $n$ copies of $K_{2}$; see Figure 5. Since $Q_{1}=K_{2}, \operatorname{dim} Q_{1}=1$. Since $Q_{2}=K_{2,2}=P_{4}, \operatorname{dim} Q_{2}=2$.
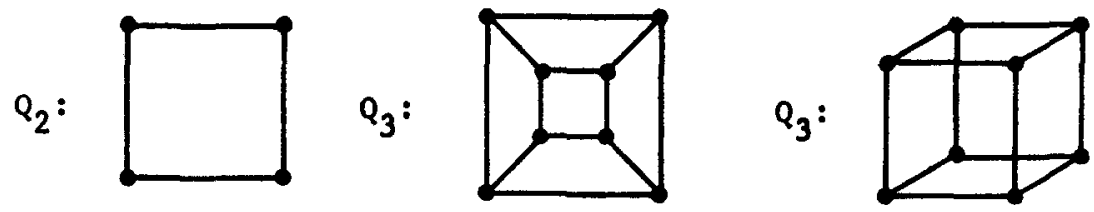

Fig. 5.

The 3-cube $Q_{3}$ is drawn twice in Figure 5. Its first appearance might suggest that its dimension is 3 . But its second depiction (in which two pairs of edges intersect) shows that $\operatorname{dim} Q_{3}=2$. Similarly, for all $n>1$, $\operatorname{dim} Q_{n}=2$.

A modest generalization of this observation asserts that for any graph $G$, $\operatorname{dim}\left(G \times K_{2}\right)$ equals $\operatorname{dim} G$, if $\operatorname{dim} G \geqslant 2$, and equals $\operatorname{dim} G+1$, if $\operatorname{dim} G=0$ or 1 .

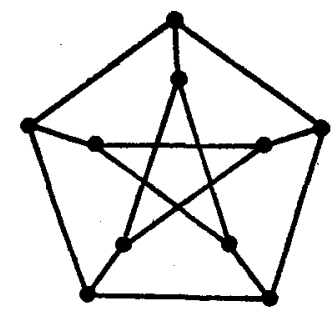

Fig. 6.

The well-known Petersen graph is shown in Figure 6. What is its dimension? It is easy to see (especially after seeing it) that the answer is 2 ; see Figure 7.

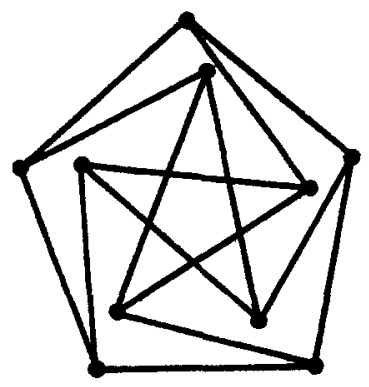

Fig. 7. 
By the way, note that the dimension of any tree is at most 2. A cactus is a graph in which no edge is on more than one polygon. Since the definition of $\operatorname{dim} G$ allows edges to intersect, it is easily seen that the dimension of any cactus is at most 2 .

In this section we have evaluated the dimension of a few special graphs. But for a given graph $G$, we know of no systematic method for determining the number $\operatorname{dim} G$. Thus the calculation of the dimension of a given graph is at present in the nature of mathematical recreation.

2. Some theorems on dimension. In the theorems of this section we use the following concepts: the girth of a graph, the chromatic number of a graph, and the chromatic number of a Euclidean space. The girth of a graph $G$ is the number of edges in its smallest polygon (if any). The chromatic number $\chi(G)$ of $G$ is the least integer $n$ such that the vertices of $G$ can be coloured using $n$ colours so that no two adjacent vertices have the same colour. The chromatic number $\chi\left(E_{n}\right)$ of a Euclidean space $E_{n}$ is the smallest number of point sets into which $E_{n}$ can be partitioned so that in no set does the distance 1 occur.

Theorem 1. For any graph $G, \operatorname{dim} G \leqslant 2 \chi(G)$.

The proof of this theorem is a simple generalization of the argument used in $\$ 1$ to establish that $\operatorname{dim} K_{m, n} \leqslant 4$; see [2]. The next two theorems do not deal with the dimension of a graph, but will be used in later proofs.

TheOREM 2. (Erdös [1]). There exists a graph with arbitrarily high girth and arbitrarily high chromatic number.

Theorem 3. (Erdös [4]). If $G$ is a graph with $n$ vertices and girth greater than $C \log n$, for $C$ sufficiently large, then $\chi(G) \leqslant 3$.

Corollary. Under the above hypothesis, $\operatorname{dim} G \leqslant 6$.

It is possible that the above hypothesis implies $\operatorname{dim} G \leqslant 3$ or even $\operatorname{dim} G \leqslant 2$, but we could not decide this question.

Theorem 4. (Erdös [3]). Among all graphs with $n$ vertices, $q$ edges, and dimension $2 k$ or $2 k+1$,

$$
\lim _{n=\infty} \max \frac{q}{n^{2}}=\frac{1}{2}\left(1-\frac{1}{k}\right)
$$

The following question was posed by Erdös [2]: What is the maximum number of edges among all graphs of dimension $d$ which have $n$ vertices? The next theorem gives the answer for $d=4$.

Theorem 5. (Erdös, unpublished). Among any $n$ points of $E_{4}$ the distance 1 between pairs of points can occur at most $n+\left[n^{2} / 4\right]$ times, and this number can be realized if $n \equiv 0(\bmod 8)$. 
We now turn to some results concerning the chromatic number of a Euclidean space. The brothers Moser [6] called for a proof of the inequality $\chi\left(E_{2}\right)>3$. Hadwiger [5] found the following inequalities.

Theorem $6 . \quad 4 \leqslant \chi\left(E_{2}\right) \leqslant 7$.

Corollary. If $\operatorname{dim} G=2$, then $\chi(G) \leqslant 7$.

Klee (unpublished) proved the next theorem.

Theorem 7. For every positive integer $n, \chi\left(E_{n}\right)$ is finite.

This result has some consequences for the dimension of a graph, but they are not as sharp as Theorem 1.

Corollary 1. If $\operatorname{dim} G$ is large, so is $\chi(G)$.

COROLlary 2. There exist graphs with arbitrarily high dimension and girth.

One might think that a graph of sufficiently high dimension must contain a complete subgraph $K_{n}$ of specified order $n>2$. That this is not necessarily so follows from the last corollary.

Unsolved problems.

I. Call a graph $G$ critical of dimension $n$ if $\operatorname{dim} G=n$ and for any proper subgraph $H, \operatorname{dim} H<n$. For example, $K_{n+1}$ is critical of dimension $n$. Characterize the critical $n$-dimensional graphs, at least for $n=3$ (this is trivial for $n=2$ ).

II. Let $G$ have $n$ vertices and assume that every subgraph $H$ with $k$ vertices has dimension at most $m$. How large can $\operatorname{dim} G$ be? (For chromatic number instead of dimension, Erdös investigates this in [4].)

\section{References}

1. P. Erdös, "Graph theory and probability", Canad. J. Math., 11 (1959), 34-38.

2. P. Erdös, "On sets of distances of $n$ points in Euclidean space", Publ. Math. Inst. Hung. Acad. Sci., 5 (1960), 165-169.

3. P. Erdös, "Some unsolved problems", Publ. Math. Inst. Hung. Acad. Sci., 6 (1961), 221-254, esp. p. 244.

4. P. Erdös, " On circuits and subgraphs of chromatic graphs ", Mathematika, 9 (1962), 170-175.

5. H. Hadwiger, " Ungelöste Probleme No. 40 ”, Elemente der Math., 16 (1961), 103-104.

6. L. Moser and W. Moser, "Solution to Problem 10", Canad. Math. Bull., 4 (1961), 187-189.

Mathematical Institute, Budapest, Hungary.

University of Michigan, Ann Arbor, U.S.A.

University of Waterloo, Waterloo, Canada. 